\title{
On the evolution of the resonant planetary system HD 128311
}

\author{
Zs. Sándor ${ }^{1,2}$ and W. Kley ${ }^{2}$ \\ 1 Department of Astronomy, Eötvös University, Pázmány Péter sétány 1/A, 1117 Budapest, Hungary \\ e-mail: Zs.Sandor@astro.elte.hu \\ 2 Institut für Astronomie und Astrophysik, Abt. Computational Physics, Universität Tübingen, Auf der Morgenstelle 10, \\ 72076 Tübingen, Germany \\ e-mail: kley@tat.physik.uni-tuebingen.de
}

Received 13 March 2006 / Accepted 23 March 2006

\begin{abstract}
Context. A significant number of the known multiple exoplanetary systems contain a pair of giant planets engaged in low-order mean-motion resonance. Such a resonant condition protects the dynamics of these planets resulting in very stable orbits. According to recent studies, this capture into a resonance is the result of a planetary migration process induced by interaction of the planets with a protoplanetary disk. If the migration is slow enough (adiabatic) near a mean motion resonance, the two planets will also be in apsidal corotation.

Aims. The recently refined orbital parameters of the system HD 128311 suggest that the two giant planets are in a 2:1 mean motion resonance, however, without exhibiting apsidal corotation. Thus the evolution of this system cannot be described by an adiabatic migration process alone. We present possible evolution scenarios of this system by combining migration processes and sudden perturbations.

Methods. We model migration scenarios through numerical integration of the gravitational $N$-body problem with additional nonconservative forces. Planet-planet scattering has been investigated by $N$-body simulations.

Results. We show that the present dynamical state of the system HD 128311 may be explained by such evolutionary processes.
\end{abstract}

Key words. planets and satellites: formation - celestial mechanics - methods: $N$-body simulations

\section{Introduction}

Among the 19 multi-planet systems found to date, about a third are engaged in low-order mean motion resonance (Vogt et al. 2005). The most prominent case is the exact $2: 1$ resonance of the two outer planets in GJ 876. There the orbital elements are determined very well, due to the short periods of the planets of $\approx 30$ and 60 days (Laughlin et al. 2005). The formation of resonant configurations between planets must be due to dissipative processes altering the semi-major axis. The orbits are in apsidal corotation for the system GJ 876, and both resonant angles librate with small amplitudes, a condition that can be explained best by a sufficiently slow and long-lasting differential migration process induced by the interaction of the planets with a protoplanetary disk (Snellgrove et al. 2001; Lee \& Peale 2002; Kley et al. 2005). Hence, the occurrence of resonances constitutes a very strong indication of migration in young planetary systems, in addition to the hot Jupiter cases.

Recent analysis of the planetary system HD 128311 suggests that two giant planets are engulfed in a 2:1 mean motion resonance, although without exhibiting apsidal corotation (Vogt et al. 2005). On the other hand, according to Lee \& Peale (2002) and Beaugé et al. (2006), the resonant planets should also be in apsidal corotation in the case of a sufficiently slow migration process. In order to resolve the above discrepancy, we construct mixed evolutionary scenarios of migrating planetary systems by incorporating migration and other additional perturbative effects. For our investigation we use $N$-body numerical integrations also containing non-conservative forces (Lee \& Peale 2002), which have been tested on full hydrodynamic evolutions of embedded planets (Kley et al. 2004). In this letter we report our findings from modeling the behavior of the resonant exoplanetary system HD 128311.

\section{Orbital solution and its stability}

Vogt et al. (2005) mention that the best fit to the radial velocity curve of HD 128311, based only on the use of Keplerian ellipses, leads to orbital data that result in unstable behavior. Therefore they also present an alternate fit for HD 128311 calculated by using three-body gravitational interactions. The orbital data determined by this alternate fit (listed in Table 1) result in stable orbits, where the two giants are in a protecting 2:1 mean motion resonance (MMR). We perform three-body numerical integrations using these initial conditions of Vogt et al. (2005), as listed in Table 1 . Only the resonant angle $\Theta_{1}=2 \lambda_{2}-\lambda_{1}-\varpi_{1}$ librates around $0^{\circ}$ with an amplitude of $\sim 60^{\circ}$ (Fig. 1, top), while both $\Theta_{2}=2 \lambda_{2}-\lambda_{1}-\varpi_{2}$ and $\Delta \varpi=\varpi_{2}-\varpi_{1}$ circulate. Here $\lambda_{\mathrm{j}}$ are mean longitudes and $\varpi_{\mathrm{j}}$ are longitudes of periapse, both numbered from the inside out. This implies that, although engaged in a MMR, the systems is not in apsidal corotation. Moreover, the eccentricities also show large oscillations (Fig. 1, bottom).

As described by Vogt et al. (2005), there exist a large variety of dynamically distinct stable orbital solutions due to the relatively high stellar jitter, resulting in the current radial velocity curve. The authors present the results obtained by a selfconsistent two-planet model (taking the mutual gravitational interactions into account) and by a Monte-Carlo procedure. 
Table 1. Orbital data of HD 128311 as provided by Vogt et al. (2005). $M$ denotes the mean anomaly.

\begin{tabular}{cccccc}
\hline \hline Planet & Mass $\left[M_{\mathrm{J}}\right]$ & $a[\mathrm{AU}]$ & $e$ & $M[\mathrm{deg}]$ & $\varpi[\mathrm{deg}]$ \\
\hline $\mathrm{b}$ & 1.56 & 1.109 & 0.38 & 257.6 & 80.1 \\
$\mathrm{c}$ & 3.08 & 1.735 & 0.21 & 166.0 & 21.6 \\
\hline
\end{tabular}

As the final outcome, they have found that only very few samples exhibit stable behavior without resonance. The remaining stable solutions are in 2:1 MMR, and in the majority of the cases $(\sim 60 \%)$ only $\Theta_{1}$ librates, while $\Theta_{2}$ circulates; in the remaining cases $\Theta_{2}$ librates as well. From this, we assume that their derived parameters in Table 1 are an accurate representation of the dynamics of the system.

\section{Evolution through a migration process}

\subsection{Adiabatic migration}

As shown in the previous section, the planets in the system HD 128311 are presently engaged in a stable 2:1 MMR. Although the actual orbital parameters do not exhibit apsidal corotation, the existence of the resonance suggests that the system has gone through a migration process in the past.

The migration of a single planet can be characterized by the migration rate $\dot{a} / a$ and the eccentricity damping rate $\dot{e} / e$. Here, we use the corresponding $e$-folding times for the semi-major axes and eccentricities: $\tau_{a}$ and $\tau_{e}$, respectively. The relation between the damping rates and $e$-folding times is $\dot{a} / a=-1 / \tau_{a}$, as for the eccentricities. Investigating the system GJ 876, Lee \& Peale (2002) have found that, for a sufficiently slow migration, the final state of the system only depends on the ratio $K$ of the $e$-folding times $K=\tau_{a} / \tau_{e}$.

From hydrodynamical calculations we know that the order of magnitude of $K$ is close to unity reflecting the physical properties (i.e. mass and viscosity) of the protoplanetary disk (Kley et al. 2004). If a system is subject to such adiabatic migration, a given value of $K$ results in unique values of the final eccentricities (Lee \& Peale 2002). However, as shown above, the eccentricities in the case of HD 128311 vary with quite large amplitudes.

We have performed a set of numerical integrations of the general co-planar three-body problem adding non-conservative drag forces, varying the value of $K$.

In these simulations the planets originally move in quasicircular orbits, and we start them from $a_{1}=4 \mathrm{AU}$ and $a_{2}=$ 2 AU. Assuming that only the outer planet is forced to migrate inward, we implement a dissipative force that results in a migration characterized by the $e$-folding time $\tau_{a}=2 \times 10^{3}$ years in the semi-major axis and $\tau_{e}=4 \times 10^{2}$ years in the eccentricity of the outer planet (corresponding to $K=5$ ). In order to obtain the present values of the semi-major axes, we slow the migration down beginning from $t_{1}=2 \times 10^{3}$ years and decreasing it linearly to zero until $t_{2}=3 \times 10^{3}$ years. In this way we model the smooth dispersal of the protoplanetary disk. After this migration process, the system is locked into a deep 2:1 resonance, while the resonant angles $\Theta_{1,2}$ and $\Delta \varpi$ librate around $0^{\circ}$ with small amplitudes. The eccentricities $e_{1}$ and $e_{2}$ are almost constant (showing only small amplitude oscillations), where the index 1 refers to the inner and index 2 to the outer planet. For the chosen $K=5$, we find $e_{1}=0.46$ and $e_{2}=0.15$, see Fig. 2. Smaller/larger values of $K$ always yield systems that are deep in resonance and result in larger/smaller $e_{1,2}$, contradicting Fig. 1.
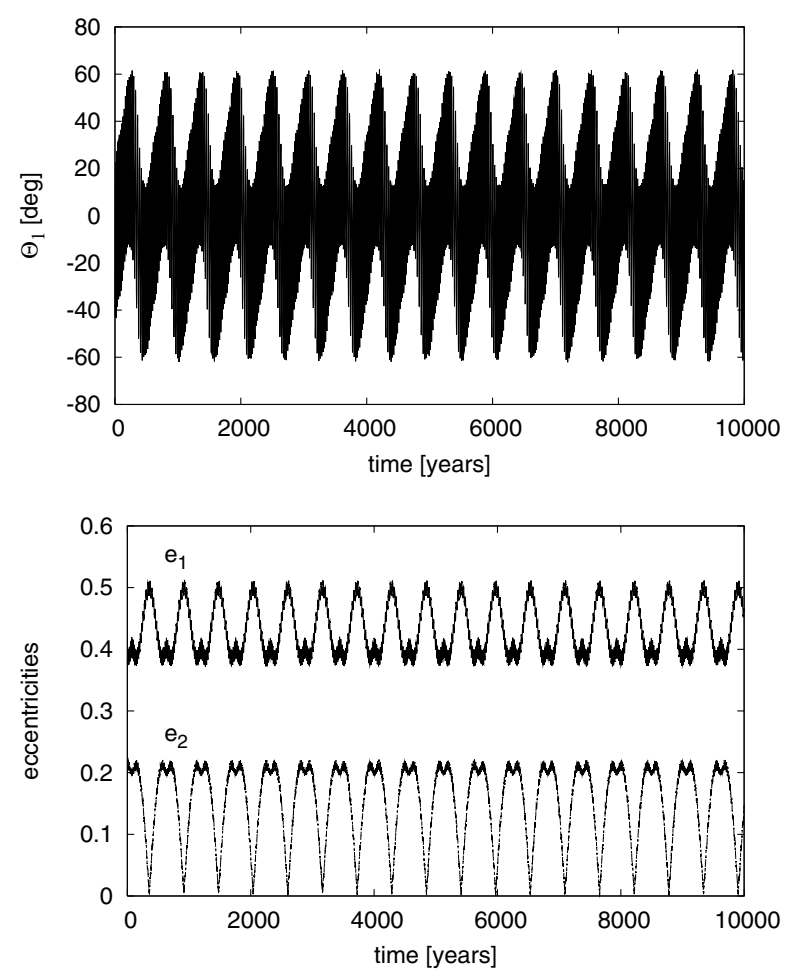

Fig. 1. Top: time evolution of the resonant angle $\Theta_{1}$ obtained by numerical integration using the initial conditions of Table 1. Bottom: the corresponding evolution of the eccentricities.

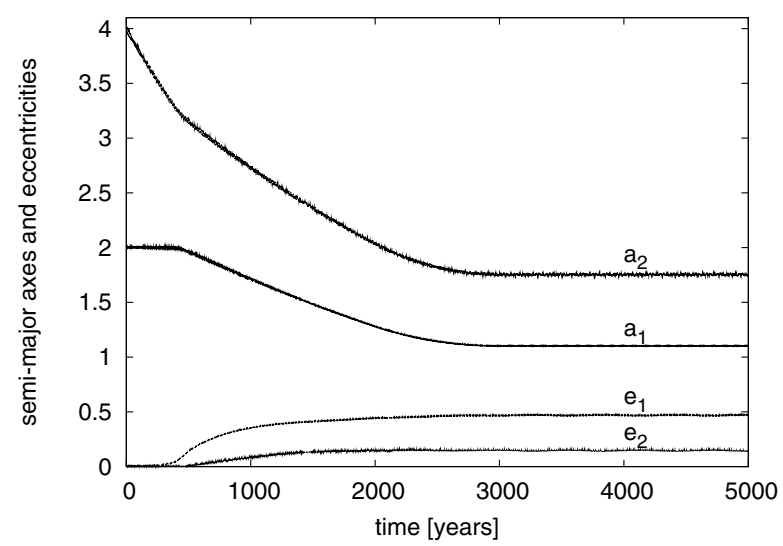

Fig. 2. The behavior of the semi-major axes and eccentricities during an adiabatic migration with $\tau_{a}=2 \times 10^{3}$ years and $K=5$. The migration is stopped between $2 \times 10^{3}$ and $3 \times 10^{3}$ years by applying a linear reduction.

Clearly, the present behavior of HD 128311 is not the result of such an adiabatic migration process alone. In the following, we present two additional mechanisms that may be responsible for the large oscillations of the eccentricities and for breaking the apsidal corotation.

\subsection{Sudden stop of the outer planet's migration}

Recent Spitzer observations of young stars confirm that the inner part of the protoplanetary disk may contain only very little mass (D'Alessio et al. 2005; Calvet et al. 2005), possibly due to photoevaporation induced by the central star. Thus, upon approaching the inner rim of such a disk, the inward migration of a planet can be terminated rapidly. 


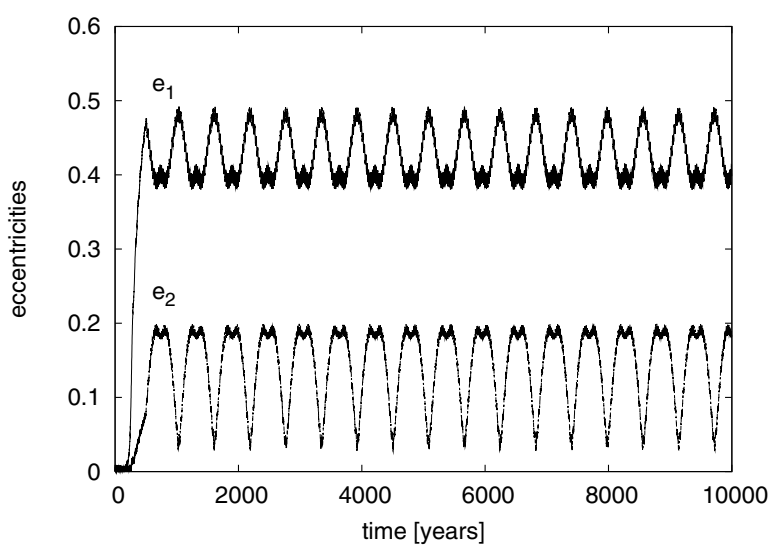

Fig. 3. The behavior of the eccentricities obtained by suddenly stopping the migration of the outer planet.

In order to model this type of scenario, we perform additional simulations where the migration of the outer planet has been stopped abruptly, reaching the actual value of its semimajor axis $\left(a_{2}=1.73 \mathrm{AU}\right)$. We assume that the inner planet orbits in the empty region of the disk at $a_{1}=1.5 \mathrm{AU}$, and then start the outer planet from $a_{2}=4 \mathrm{AU}$, forcing it to migrate inward very fast $\left(\tau_{a}=500\right.$ years). We find that the present behavior of the eccentricities can be obtained by using $K=10$. The sudden stop of the migration results in a behavior of the eccentricities, as shown in Fig. 3, that is very similar to the observed case (Fig. 1). We note that the planets remain in apsidal corotation after the above sudden stop of the migration but with increased libration amplitudes of the resonant angles.

\subsection{Planet-planet scattering event}

The behavior of the eccentricities of the giant planets in the system HD 128311 is very similar to what is observed in the system around $v$ Andromedae. Ford et al. (2005) propose that such a behavior in $v$ And is most likely the result of a planet-planet scattering event. When investigating the resonant system HD 73526 , Tinney et al. (2006) also suggest that the lack of apsidal corotation can be the result of a dynamical scattering event. Therefore we investigate whether the present behavior of HD 128311 can be modeled by this effect and present two cases.

\section{Additional inner planet}

In this case we assume that an additional small mass planet is orbiting close to the central star in a quasi circular orbit. When the outer giants are far enough from the star, the orbit of the low mass planet is stable. However, as the giants migrate inward and approach their present positions, they perturb the orbit of the small planet thereby making its motion chaotic, which in long term may increase its eccentricity. Finally, due to its high eccentricity, the small planet can suffer a close encounter with one of the giant planets. After the close encounter the small planet may be ejected from the system or pushed into an orbit with a very large semi-major axis. Depending on the initial position of the small planet, it can also be captured into a mean-motion resonance with the inner giant and forced to migrate inward. During the migration its eccentricity will increase on a much shorter timescale than by a "pure" chaotic evolution. Thus, in this case a close encounter with the inner giant is very likely as well.
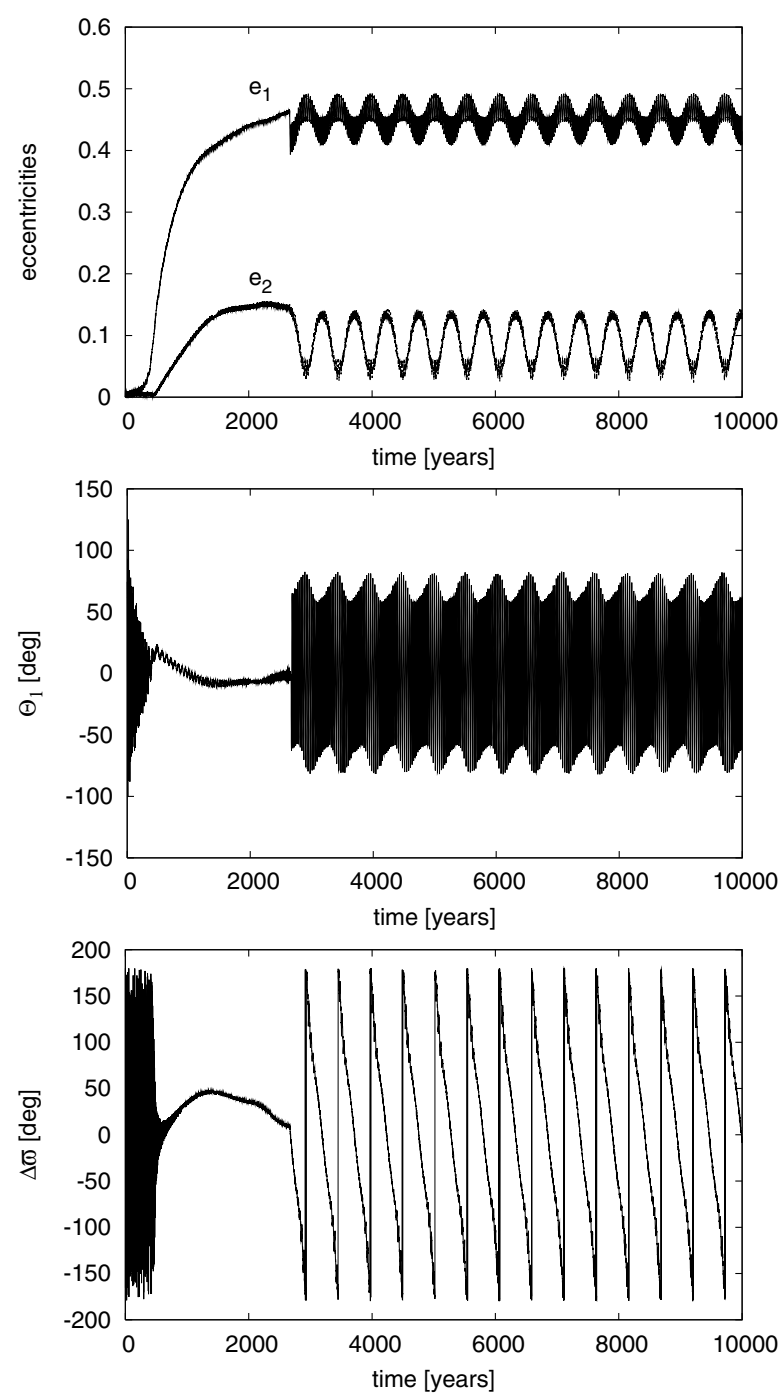

Fig. 4. Time evolution of the eccentricities (top), resonant angles $\Theta_{1}$ (middle) and $\Delta \varpi$ (bottom) of the inward migrating giant planets, before and after a scattering event with an inner low mass planet.

In Fig. 4 we show the behavior of the eccentricities and the resonant angles $\Theta_{1}$ and $\Delta \varpi$ when the giant planets are migrating inwards with initial conditions given in Sect. 3.1. Starting the small planet $\left(m=0.03 M_{\mathrm{Jup}}\right)$ from a nearly circular orbit with $a=0.5 \mathrm{AU}$, it is captured into a resonance with the inner giant. During the migration the small planet's eccentricity increases finally so that it crosses the orbits of giant planets and suffers a close encounter with them. After the encounter at $t \approx 2500 \mathrm{yr}$, the apsidal corotation of the giant planets breaks (i.e. $\Theta_{2}$ and $\Delta \varpi$ circulate), but the giant planets remain in the 2:1 resonance, since $\Theta_{1}$ still librates around $0^{\circ}$, see Fig. 4.

\section{Additional outer planet}

In this second case we assume that the third planet $(m=$ $0.03 M_{\mathrm{Jup}}$ ) originates from the outer region and that it approaches the giant planets through inward migration, where we assume that the adiabatic migration of the two giant planets is already finished, as displayed in Fig. 2. They orbit, for instance, in a gas-free environment, while the small planet is still embedded in the outer protoplanetary disk. In Fig. 5 we display the variation in the eccentricities of the giants after the scattering event. 


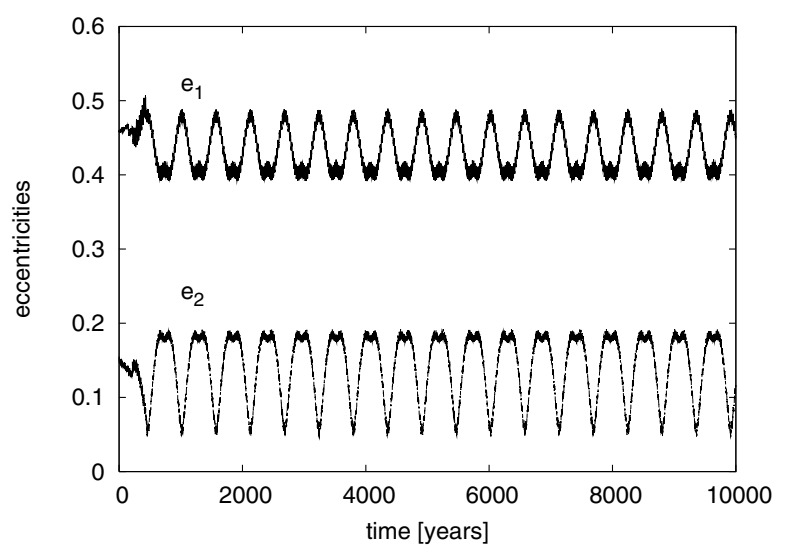

Fig. 5. The behavior of the eccentricities before and after a scattering with a small mass planet migrating inward from outside starting from $a=2.6 \mathrm{AU}$.

The small planet is started from $a=2.6 \mathrm{AU}$ and migrates inward with an $e$-folding time of $\tau_{a}=2 \times 10^{3}$ years. After scattering it is pushed into a very distant orbit $a \sim 300 \mathrm{AU}$. We note that in this case the giant planets remain in apsidal corotation, but with a substantial increase in $\Theta_{2}$ and $\Delta \varpi$. In additional simulations, we find that apsidal resonance is always preserved; however, we cannot exclude the possibility of breaking the apsidal corotation entirely.

\section{Conclusions}

In this paper we have investigated evolutionary scenarios that may have led to the observed behavior of the resonant system HD 128311, where the two planets are engaged in a 2:1 mean motion resonance but not in apsidal corotation. We assumed that the two giant planets were formed far from the central star and migrated inwards, due to gravitational interaction with the protoplanetary disk. During this differential migration, they were locked into the 2:1 mean motion resonance. At the end of their migration we imagined that the system suffered a sudden perturbation.

As a first case we studied the sudden stop of the migration, which may be induced by an inner rim of the disk and an empty region inside of it, as indicated by some observations of young stars.

In the second scenario the sudden perturbation is caused by a planet-planet scattering event, similar to the one suggested by Ford et al. (2005) in the case of $v$ And. We analyzed an encounter with a small $\left(\sim 10 M_{\oplus}\right)$ planet, approaching the two massive planets either from outside or inside, which was thrown then into a large $a$ orbit or directly ejected after the scattering event.

We find that the sudden perturbation caused by an encounter with an inner small planet is clearly able to break the apsidal resonance of the two planets. But also in the other cases, the orbital behavior of the giants is very similar to the most recent orbital data of HD 128311 (Vogt et al. 2005).

The system HD 128311 constitutes another example that demonstrates the important interplay of migration and scattering processes in shaping the dynamics of exoplanetary systems.

Acknowledgements. The authors acknowledge the supports of the Hungarian Scientific Research Fund (OTKA) under the grant D048424 and the German Science Foundation (DFG) under grant 436 UNG 17/5/05. W.K. would like to thank Greg Laughlin for the many fruitful discussions.

\section{References}

Beaugé, C., Michtchenko, T. A., \& Ferraz-Mello, S. 2006, MNRAS, 365, 1160 Calvet, N., D'Alessio, P., Watson, D. M., et al. 2005, ApJ, 630, L185 D'Alessio, P., Hartmann, L., Calvet, N., et al. 2005, ApJ, 621, 461 Ford, E. B., Lystad, V., \& Rasio, F. A. 2005, Nature, 434, 873 Kley, W., Peitz, J., \& Bryden, G. 2004, A\&A, 414, 735

Kley, W., Lee, M. H., Murray, N., \& Peale, S. J. 2005, A\&A, 437, 727 Laughlin, G., Butler, R. P., Fischer, D. A., et al. 2005, ApJ, 622, 1182 Lee, M. H., \& Peale, S. J. 2002, ApJ, 567, 596

Snellgrove, M. D., Papaloizou, J. C. B., \& Nelson, R. P. 2001, A\&A, 374, 1092 Tinney, C. G., Butler, R. P., Marcy, G. W., et al. 2006, [arXiv: astro-ph/0602557] Vogt, S. S., Butler, R. P., Marcy, G. W., et al. 2005, ApJ, 632, 638 